TAO, Vol. 15, No. 1, 89-96, March 2004

NOTES AND CORRESPONDENCE

\title{
Error Analysis of Retrieved Aerosol Optical Depth and Surface Reflectance due to the Azimuthal Angle Simplification of Atmospheric Functions
}

\author{
Chien-Hui Liu ${ }^{1, *}$ \\ (Manuscript received 1 April 2003, in final form 2 December 2003)
}

\begin{abstract}
Using a simplified azimuthal angle assumption of atmospheric functions in the atmospheric correction model, such as ATmospheric CORrection model version 2 (ATCOR2) and ATmospheric CORrection model version 3 (ATCOR3) developed by Richter (1997) for Systeme Pour l'Observation de la Terre (SPOT) High Resolution Visible (HRV) data, can generate errors. This paper discusses the errors of retrieved surface reflectance and aerosol optical depth (AOD) due to such simplification. The results indicate that the relative error of retrieved surface reflectance can exceed $40 \%$ in the forward-scattering direction in hazy sky and that of retrieved AOD can be over $10 \%$ in the anti-solar direction in clear sky at the extreme view angle. Hence, the consideration of azimuthal angle dependence of atmospheric functions is indispensable for the atmospheric correction model of SPOT HRV data, particularly in large view angles, e.g., larger than $\mathbf{- 2 1 . 5 ^ { \circ }}$ (backward-scattering region) and $24.9^{\circ}$ (forward-scattering region) in clear sky, and larger than $-19.1^{\circ}$ and $12.9^{\circ}$ in hazy sky.
\end{abstract}

(Key words: Aerosol optical depth, Surface reflectance, SPOT)

\section{INTRODUCTION}

The importance of the atmospheric correction of remotely sensed data is now broadly

1 Department of Information Management/Geographic Information Management and Research Center, Transworld Institute of Technology, Douliu, Taiwan, ROC

* Corresponding author address. Chien-Hui Liu, Department of Information Management/Geographic Information Management and Research Center, Transworld Institute of Technology, Douliu, Yunlin 64045, Taiwan, ROC; E-mail:chliu@tit.edu.tw 
known, since satellite sensors receive signals not only by reflection from a target but also by atmospheric scattering and absorption. Multi-temporal atmospheric corrected satellite images can monitor crop growth, improve land-use classification, and detect change of the environment more efficiently than can raw data.

The atmospheric effects of remotely sensed data include both molecular and aerosol absorption and scattering (Liang et al. 1997). Atmospheric window channels are used to minimize the gaseous absorption, especially for water vapor and ozone. Because the concentrations of ozone and oxygen are quite stable both in time and space, their absorption and scattering are relatively easy to correct. Radiosonde or climatological data can be used to estimate water vapor content and its absorption. Owing to the nature of temporal and spatial variation of aerosols, the scattering and absorption of aerosols are the main variables in the atmospheric correction of remotely sensed data.

Radiative transfer theory is the basic principle of the atmospheric correction method (Liang et al. 1997). If molecular absorption can be de-coupled from scattering, top-of-atmosphere (TOA) reflectance can be determined from atmospheric reflectance, total transmission (sum of both direct and diffuse transmissions) of the atmosphere, spherical albedo of the atmosphere and gas absorption (see Vermote et al. 1997b for details). These atmospheric functions, which are defined as the functions to simulate TOA reflectance and correct atmospheric effect of remotely sensed data, can be calculated by using radiative transfer (RT) codes. The Second Simulation of the Satellite Signal in the Solar Spectrum (6S) (Vermote et al. 1997b) and the MODerate resolution TRANSsmittance (MODTRAN) (Berk et al. 1989; Berk et al. 1998) are validated RT codes for determining these atmospheric functions, which can be stored as lookup tables and used to develop atmospheric correction algorithms (Liang et al. 1997; Vermote et al. 1997a; El Saleous et al. 2000). The advantage of the look-up tables is the repeated use of the time-consuming results of the RT computation. Therefore, the accuracies of retrieved aerosol optical depth (AOD) and surface reflectance strongly depend on the accuracies of the look-up tables of the atmospheric functions.

The look-up tables of the atmospheric functions should cover the wide range of, not only atmospheric conditions (Richter 1997), but also solar and satellite viewing geometries. Vermote and Vermeulen (1999) and El Saleous et al. (2000) determined the look-up tables of the atmospheric functions for Earth Observing System - MODerate resolution Imaging Spectroradiometer (EOS-MODIS) and National Oceanic and Atmospheric Administration - Advanced Very High Resolution Radiometers (NOAA-AVHRR) sensor, respectively. The errors of the simulated TOA reflectances were small $(\sim 0.001)$ and negligible. Among the atmospheric functions (atmospheric reflectance, diffuse transmittance and spherical albedo), atmospheric reflectance was computed at, not only several solar zenith angles, view zenith angles and AODs, but also some (73) relative azimuth angles. In fact, atmospheric reflectance is dependent on relative azimuthal angle by radiative transfer theory (Liou 1981). However, ATmospheric CORrection model version 2 (ATCOR2) and ATmospheric CORrection model version 3 (ATCOR3) (Richter 1996a; Richter 1996b), which have been commercialized (Erdas Imagine, http://www.erdas. com/; PCI Geomatica, http://www.pcigeomatics.com/), simplify the azimuthal dependence of atmospheric functions (using only at relative azimuthal angles of 30 and 150 degrees) for Systeme Pour l'Observation de la Terre (SPOT) images in order to keep down the size and 
then reduce the computational time (CPU time) of the lookup tables. SPOT satellite scans multi-spectral images with spatial resolution of 20 meters and covers ground swath widths of $60 \mathrm{~km}$. The reduction of CPU time can be as a factor of 33.3 for wide ranges of solar zenith angle, viewing zenith angle and AOD. Although they have been utilized in the monitoring of boreal forest in Siberia (Petelina 1998), the development of permafrost in emerging marine sediments near the eastern shore of Hudson Bay, Canada (King 2000) and the monitoring of urban land cover change in Phoenix of Arizona, USA (Stefanov et al. 2001), using Landsat Thematic Mapper (TM) images, few of the results using SPOT images have been published. Furthermore, these researchers, including Richter (1996a, 1996b and 1997) and Richter et al. (2002), had no field measurements to assess the errors of rerieved AOD and surface reflectance. Consequently, it's worth studying the possible errors due to the simplifications in ATCOR2 and ATCOR3.

This paper assesses the errors of retrieved AOD and surface reflectance due to the simplified consideration of azimuthal angle of the atmospheric functions by ATCOR2 and ATCOR3. The $6 \mathrm{~S}$ code is used forwardly to simulate the TOA reflectances and inversely to estimate these errors. Although the MODTRAN2 code is implemented to compile the look-up tables of the atmospheric functions for ATCOR2 and ATCOR3 (Richter 1996a, 1996b and 1997), the 6S and MODTRAN codes all use sophisticated radiative transfer methods, such as successive orders of scattering and discrete ordinate methods (Berk et al. 1998), respectively, to account for multiple scattering of the atmosphere. Therefore, both codes are generally accepted and validated models for the purpose of developing an atmospheric correction model (Richter et al. 2002). In this study, an experiment is conducted to estimate the possible errors due to the simplified consideration by ATCOR 2 and ATCOR3 and then to emphasize the necessity of the azimuthal angle dependence of the atmospheric functions in the development of an atmospheric correction model.

\section{METHODOLOGY}

The 6S code (Vermote et al. 1997b) is used to simulate TOA reflectance $\rho_{T O A}$ for various AOD and surface reflectances. It can determine $\rho_{T O A}$ (and also the atmospheric functions) by using several input parameters, such as solar illumination and satellite observation geometries (zenith and azimuthal angles), atmospheric model (tropical, mid-latitude summer, mid-latitude winter, sub-arctic summer, sub-artic winter and US62 atmospheres), aerosol model (e.g., continental, urban, maritime and desert), aerosol concentration (optical depth) or visibility, altitudes of sensor and target, sensor response function and surface reflectance. $\rho_{\text {TOA }}$ can also be used as input together with all of the other known parameters, including aerosol optical depth, to obtain the surface reflectance $\rho_{s}$ under the assumption of uniform target. Aerosol optical depth $\tau_{A}$ is the best value fitted to $\rho_{T O A}$ for known (or assumed) $\rho_{s}$.

An experiment is designed to assess both the retrieved surface reflectance error $\delta \rho_{s} / \rho_{s}$ and the retrieved aerosol optical depth error $\delta \tau_{A} / \tau_{A}$, when the azimuthal dependence of atmospheric functions is not considered (as ATCOR2 and ATCOR3 considered only relative azimuthal angles $\phi$ of $30^{\circ}$ and $150^{\circ}$ ). The tropical atmospheric model is used. The reflection 
of the target is considered as Lambertian. The aerosol model is considered to be continental. The solar zenith angle $\theta_{s}$ is set to $60^{\circ}$, which is about the maximum value in a year when SPOT satellite passes Taiwan. The altitude of the target is set to $0 . \rho_{s}$ is set to 0.02 (as a dark target) in the multi-spectral band 2 (XS2), i.e. red band. $\rho_{T O A}$ is simulated at various viewing zenith angles $\theta_{v}\left(0^{\circ}\right.$ to $\left.27^{\circ}\right)$ and $\phi\left(0^{\circ}\right.$ to $\left.180^{\circ}\right)$ in clear (VIS (Visibility) $\left.=23 \mathrm{~km}, \mathrm{AOD}=0.24\right)$ and hazy (VIS $=5 \mathrm{~km}, \mathrm{AOD}=0.71)$ skies. Considering $\rho_{\text {TOA }}$ in the principal plane $\left(\phi\right.$ of $0^{\circ}$ and $180^{\circ}$ ) replaced by $\phi$ of $30^{\circ}$ and $150^{\circ}$ as simplified by ATCOR2, $\delta \rho_{s} / \rho_{s}$ and $\delta \tau_{A} / \tau_{A}$ are then estimated by using the lookup table approach with 6S (Vermote and Vermeulen 1999).

\section{RESULTS AND DISCUSSIONS}

Before discussing $\delta \tau_{A} / \tau_{A}$ and $\delta \rho_{s} / \rho_{s}$, atmospheric reflectance $\rho_{a}$ and its relative error $\delta \rho_{a} / \rho_{a}(\%)$ as a function of relative azimuthal angle $\phi$ in clear and hazy sky are depicted in Figs. la, b. $\theta_{v}$ is set to the extreme case $\left(27^{\circ}\right)$ for SPOT HRV sensor. $\delta \rho_{a} / \rho_{a}$ is defined as the error, when $\rho_{a}$ at $\phi$ from $0^{\circ}$ to $90^{\circ}$ (forward-scattering region) and those values at $\phi$ from $90^{\circ}$ to $180^{\circ}$ (backward-scattering region) are replaced by $\rho_{a}$ at $\phi$ of $30^{\circ}$ and $150^{\circ}$, respectively. The application of this definition is the same as that used for azimuthal angle simplification of atmospheric functions by ATCOR2 and ATCOR3. $\rho_{a}$ decreases with $\phi$ from $0^{\circ}$ to $97^{\circ}$, and then increases with $\phi$ to $180^{\circ}$ in clear sky (Fig. la); it shows strong forward scattering. $\delta \rho_{a} / \rho_{a}$ increases from $-2.2 \%$ to $7.3 \%$ in forward-scattering region, and decreases from $4.4 \%$ to $-1.5 \%$ in backward-scattering region. If the sky is hazy, $\rho_{a}$ decreases with $\phi$ from $0^{\circ}$ to $72^{\circ}$, and then increases with $\phi$ to $180^{\circ}$ (Fig. lb). Backward scattering is stronger than forward scattering because of the larger aerosol optical depth. $\delta \rho_{a} / \rho_{a}$ increases from $-2.4 \%$ to $4.1 \%\left(\phi=71^{\circ}\right)$ and then decreases to $2.7 \%$ in forward-scattering region, and it decreases from $17.8 \%$ to $-4.1 \%$ in backward-scattering region (Fig. lb). Certainly, such errors can result in the errors of $\rho_{\text {TOA }}$ ; the retrieved $\tau_{A}$ and $\rho_{s}$ are discussed below.

Figure 2a shows the relative error of retrieved aerosol optical depth $\delta \tau_{A} / \tau_{A}$ as a function of view angle in clear and hazy sky, when $\rho_{T O A}$ in the principal plane is replaced by those values at $\phi$ of $30^{\circ}$ and $150^{\circ} . \delta \tau_{A} / \tau_{A}$ increases with $\theta_{v}$, and can be $-11.2 \%$ at $\theta_{v}=-27^{\circ}$ and $-5.8 \%$ at $\theta_{v}=27^{\circ}$ in clear sky. Negative $\theta_{v}$ implies the backscattering region. However, those errors are reduced to $-4.6 \%$ at $\theta_{v}=-27^{\circ}$ and $-6.2 \% \theta_{v}=27^{\circ}$ in hazy sky. In fact, the absolute error of the retrieved AOD is -0.033 in hazy sky and also exceeds -0.027 in clear sky.

The relative error of retrieved surface reflectance $\delta \rho_{s} / \rho_{s}$ shows a similar pattern with $\delta \tau_{A} / \tau_{A}$ (Fig. 2b). $\delta \rho_{s} / \rho_{s}$ increases with $\theta_{v}$, and is $-15.4 \%$ at $\theta_{v}=-27^{\circ}$ and $-12.5 \%$ at $\theta_{v}=27^{\circ}$ in a clear sky. Aerosol scattering increases with $\theta_{v}$, and is stronger in the principal plane than in other azimuthal planes (strong atmospheric backscattering at $\phi$ of $0^{\circ}$ and $180^{\circ}$, as shown in Figs. la, b; therefore, the estimated $\rho_{s}$ is lower as $\theta_{v}$ increases. As VIS reduces to $5 \mathrm{~km}$ (hazy sky), $\delta \rho_{s} / \rho_{s}$ can approach to $-23.6 \%$ at $\theta_{v}=-27^{\circ}$ and $-46.8 \%$ at $\theta_{v}=27^{\circ}$. If $\delta \rho_{s} / \rho_{s}$ is required to be less than $-10 \%, \theta_{v}$ should be from $-21.5^{\circ}$ to $24.9^{\circ}$ in clear sky, and from $-19.1^{\circ}$ to $12.9^{\circ}$ in hazy sky.

The above discussions underscore the importance of considering the azimuthal 
(a)

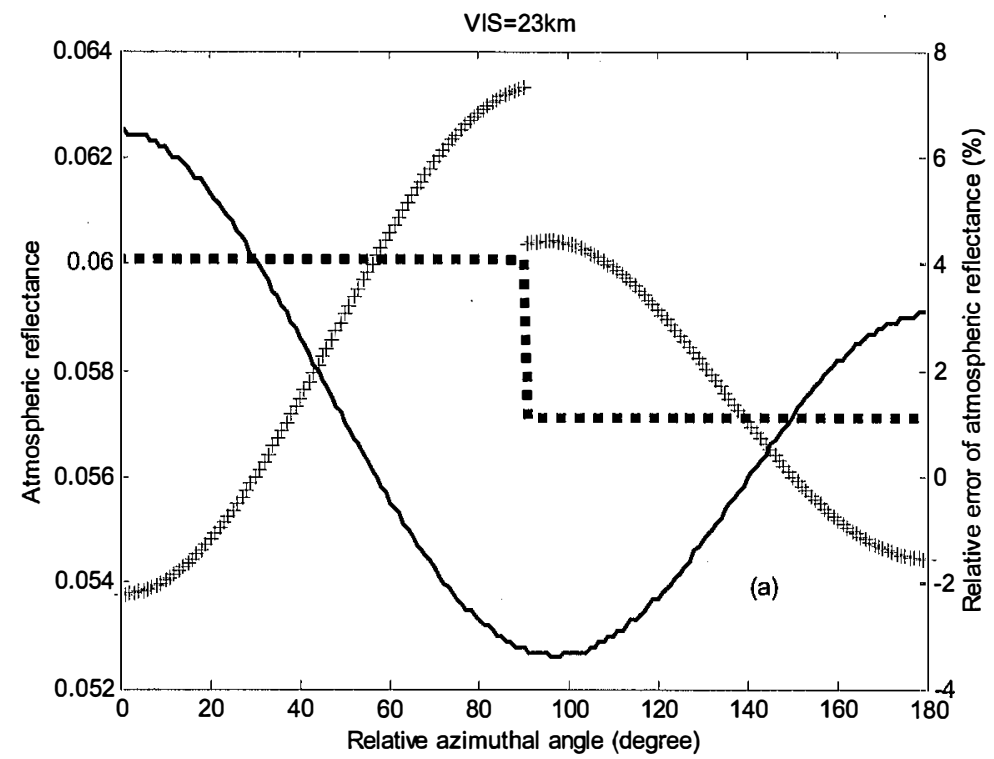

(b)

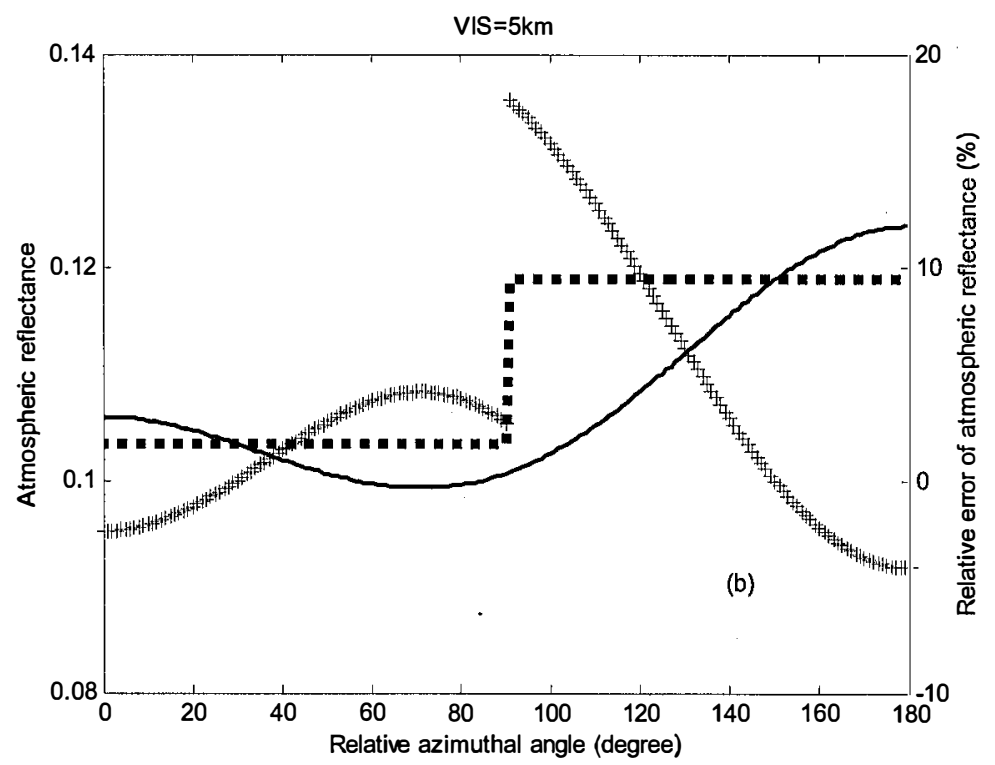

Fig. 1. Atmospheric reflectance (solid line) and its relative error (plus sign) (\%) as a function of relative azimuthal angle in (a) clear (VIS $=23 \mathrm{~km}$ ) and (b) hazy (VIS $=5 \mathrm{~km})$ sky. $\theta_{v}$ is set to $27^{\circ}$. Relative error of atmospheric reflectance is defined as if atmospheric reflectances (relative azimuthal angles from $0^{\circ}$ to $180^{\circ}$ ) are replaced by those values at $\phi$ of $30^{\circ}$ and $150^{\circ}$ (dotted line). 
(a)

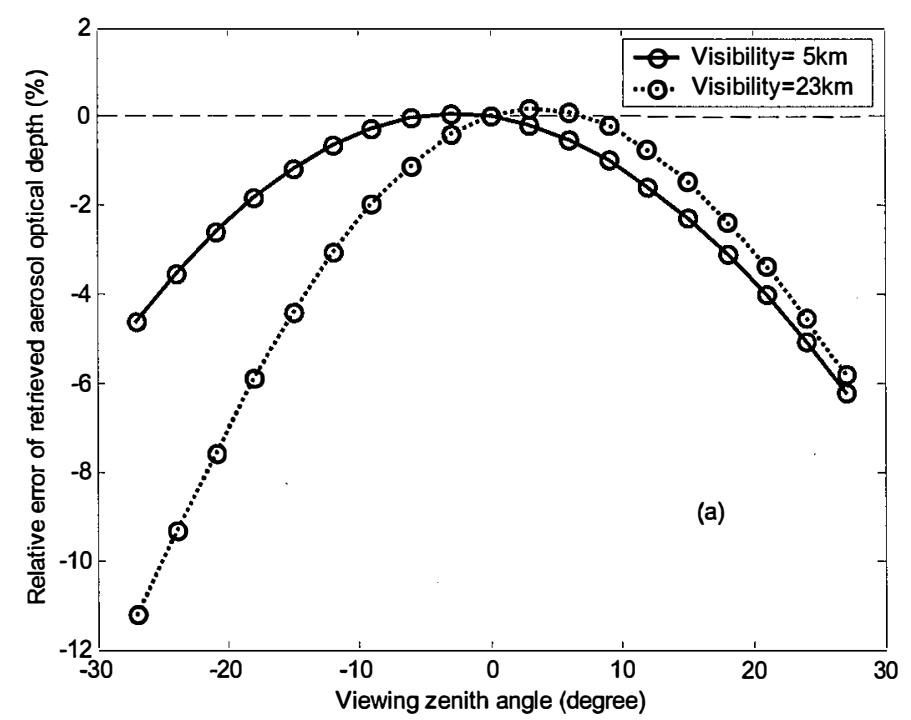

(b)

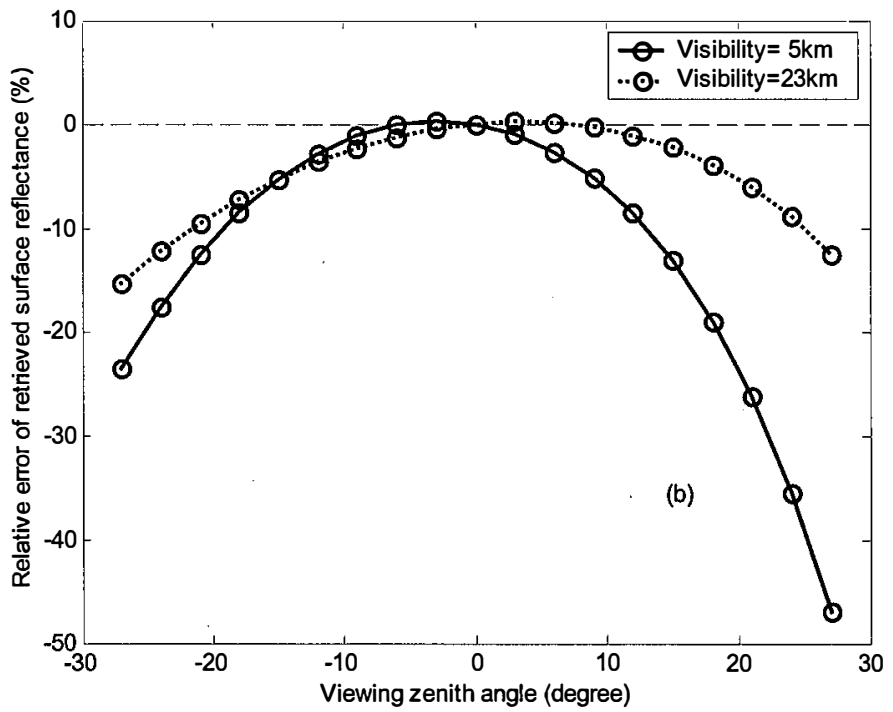

Fig. 2. (a) Relative error of retrieved aerosol optical depth (\%) and (b) relative error of retrieved surface reflectance (\%) as a function of view angle in clear $(\mathrm{VIS}=23 \mathrm{~km}$ ) and hazy (VIS $=5 \mathrm{~km})$ sky, when $\rho_{\text {TOA }}$ in the principal plane (relative azimuthal angles are $0^{\circ}$ and $180^{\circ}$ ) are replaced by those values at $\phi$ of $30^{\circ}$ and $150^{\circ} . \rho_{s}$ is set to be 0.02 and the waveband is XS2 band. Negative view angle implies the backscattering region. 
dependence of atmospheric functions in developing the atmospheric correction model. Hence, one should bear in mind the errors introduced by ATCOR2 and ATCOR3, especially for large $\theta_{v}$, e.g., $\theta_{v}$ larger than $-21.5^{\circ}$ (backward-scattering region) and $24.9^{\circ}$ (forward-scattering region) in clear sky, and larger than $-19.1^{\circ}$ and $12.9^{\circ}$ in hazy sky.

\section{CONCLUSIONS}

This paper assesses the errors of retrieved surface reflectance and AOD for SPOT HRV data due to the azimuthal angle simplification of atmospheric functions used in some commercial atmospheric correction software, such as ATCOR2 and ATCOR3. The results show that relative error of retrieved AOD can be over 10\% in the anti-solar direction in clear sky and that of retrieved surface reflectance can exceed $40 \%$ in the forward-scattering direction in hazy sky at the extreme view angle. Therefore, it's very important to appreciate the azimuthal angle dependence of atmospheric functions in the development of an atmospheric correction model of SPOT HRV data.

Acknowledgements This work is supported by the National Science Council, R.O.C., under grant NSC91-2212-E-265-001. The manuscript was greatly improved through comments from the anonymous reviewers.

\section{REFFERENCES}

Berk, A., L. S. Bernstein, and D. C. Robertson, 1989: MODTRAN: a moderate resolution model for LOWTRAN 7, GL-TR-89-0122, Geophysics Laboratory, Bedford, MA, U. S. A.

Berk, A., L. S. Bernstein, G. P. Anderson, P. K. Acharya, D. C. Robertson, J. H. Chetwynd, and S. M. Adler-Golden, 1998: MODTRAN cloud and multiple scattering upgrades with application to AVIRIS. Remote Sens. Environ., 65, 367-375.

El, Saleous, N. Z., E. F. Vermote, C. O. Justice, J. R. G. Townshend, C. J. Tucker, and S. N. Goward, 2000: Improvements in the global biospheric record from the Advanced Very High Resolution Radiometer (AVHRR). Int. J. Remote Sens., 21, 1251-1277.

Liang, S., H. Fallah-Adl, S. Kalluri, J. Jaja, Y. J. Kaufman and J. R. G. Townshend, 1997: An operational atmospheric correction algorithm for Landsat Thematic Mapper imagery over the land. J. Geophys. Res., 102, 17173-17186.

Liou, K. N., 1981: An introduction to atmospheric radiation, O-Ya, Taipei, 392pp.

King, L., 2000: Frozen ground. The News Bulletin of the International Permafrost Association, 24, December.

Petelina, S. V., 1998: Optical Model of Atmospheric Aerosols in Russian Siberia for Correction of Satellite Data. IR-98-078/October, International Institute for Applied Systems Analysis, Laxenburg, Austria. 
Richter, R., 1996a: A spatially adaptive fast atmospheric correction algorithm. Int. J. Remote Sens., 17, 1201-1214.

Richter, R., 1996b: Atmospheric correction of satellite data with haze removal including a haze/clear transition region. Comp. \& Geos., 22, 675-681.

Richter, R., 1997: Correction of atmospheric and topographic effects for high spatial resolution satellite imagery. Int. J. Remote Sens., 18, 1099-1111.

Richter, R., A. Muller, and U. Heiden, 2002: Aspects of operational atmospheric correction of hyperspectral imagery. Int. J. Remote Sens., 23, 145-157.

Stefanov, W. L., M. S. Ramsey and P. R. Christensen, 2001: Monitoring urban land cover change: An expert system approach to land cover classification of semiarid to arid urban centers. Remote Sens. Environ., 77, 173- 185.

Vermote, E. F., N. El Saleous, C. O. Justice, Y. J. Kaufman, J. L. Privette, L. Remer, J. C. Roger, and D. Tanré, 1997a: Atmospheric correction of visible to middle-infrared EOSMODIS data over land surfaces: background, operational algorithm and validation. $J$. Geophys. Res., 102, 17131-17141.

Vermote, E. F., D. Tanre, J. L. Deuze, M. Herman, and J.-J. Morcrette, 1997b: Second simulation of the satellite signal in the solar spectrum, 6S: an overview. IEEE Tran. Geosci. Remote Sens., 35, 675-686.

Vermote, E. F., and A. Vermeulen, 1999: MODIS Algorithm Technical Background Document, Atmospheric correction algorithm: spectral reflectances (MOD09) version 4.0, NASA contract NAS5-96062. 